\title{
A more accurate reverse half-discrete Hilbert-type inequality
}

\section{Aizhen Wang and Bicheng Yang*}

\section{"Correspondence:}

bcyang@gdei.edu.cn

Department of Mathematics,

Guangdong University of

Education, Guangzhou, Guangdong

510303, P.R. China

\begin{abstract}
Using the way of weight functions and the idea of introducing parameters, and by means of Hermite-Hadamard's inequality, a more accurate reverse half-discrete Hilbert-type inequality with the non-homogeneous kernel and a best constant factor is established. In addition, its best extension with parameters, the equivalent forms, as well as some particular cases are given.
\end{abstract}

MSC: 26D15; 47A07

Keywords: weight function; non-homogeneous kernel; reverse; equivalent form; Hermite-Hadamard's inequality

\section{Introduction}

If $p>1, \frac{1}{p}+\frac{1}{q}=1, a_{n}, b_{n} \geq 0, a=\left\{a_{n}\right\}_{n=1}^{\infty} \in l^{p}, b=\left\{b_{n}\right\}_{n=1}^{\infty} \in l^{q},\|a\|_{p}=\left\{\sum_{n=1}^{\infty} a_{n}^{p}\right\}^{1 / p}>0$, and $\|b\|_{q}=\left\{\sum_{n=1}^{\infty} b_{n}^{q}\right\}^{1 / q}>0$, then we have the following famous discrete Hilbert-type inequality $(c f .[1])$ :

$$
\sum_{n=1}^{\infty} \sum_{m=1}^{\infty} \frac{\ln (m / n)}{m-n} a_{m} b_{n}<\left[\frac{\pi}{\sin (\pi / p)}\right]^{2}\|a\|_{p}\|b\|_{q}
$$

where the constant factor $[\pi / \sin (\pi / p)]^{2}$ is the best possible. Moreover the integral analogue of inequality (1) is given as follows (cf. [1]): If $p>1, \frac{1}{p}+\frac{1}{q}=1, f(x), g(x) \geq 0$, $f \in L^{p}(0, \infty), g \in L^{q}(0, \infty),\|f\|_{p}=\left\{\int_{0}^{\infty} f^{p}(x) d x\right\}^{\frac{1}{p}}>0,\|g\|_{q}=\left\{\int_{0}^{\infty} g^{q}(x) d x\right\}^{\frac{1}{q}}>0$, then

$$
\int_{0}^{\infty} \int_{0}^{\infty} \frac{\ln (x / y)}{x-y} f(x) g(y) d x d y<\left[\frac{\pi}{\sin (\pi / p)}\right]^{2}\|f\|_{p}\|g\|_{q}
$$

with the same best constant factor $[\pi / \sin (\pi / p)]^{2}$.

In 2006, Yang proved the following more accurate inequality of (1) (cf. [2]): If $p>1$, $\frac{1}{p}+\frac{1}{q}=1, \frac{1}{2} \leq \alpha \leq 1, a_{n}, b_{n} \geq 0$, such that $0<\|a\|_{p}<\infty$ and $0<\|b\|_{q}<\infty$, then

$$
\sum_{n=0}^{\infty} \sum_{m=0}^{\infty} \frac{\ln \left(\frac{m+\alpha}{n+\alpha}\right)}{m-n} a_{m} b_{n}<\left[\frac{\pi}{\sin (\pi / p)}\right]^{2}\|a\|_{p}\|b\|_{q}
$$

where the constant factor $[\pi / \sin (\pi / p)]^{2}$ is still the best possible. Inequalities (1)-(3) are important in mathematical analysis and its applications [3]. There are lots of improve-

() 2015 Wang and Yang; licensee Springer. This is an Open Access article distributed under the terms of the Creative Commons Attribution License (http://creativecommons.org/licenses/by/4.0), which permits unrestricted use, distribution, and reproduction in any medium, provided the original work is properly credited. 
ments, generalizations, and applications of inequalities (1)-(3); for more details, refer to [4-12].

At present, the research on the half-discrete Hilbert-type inequalities has gradually become in focus. We find a few results on the half-discrete Hilbert-type inequalities with the non-homogeneous kernel, which were published early ( $c f$. [1], Theorem 351 and [13]). Recently, Yang gave some half-discrete Hilbert-type inequalities (cf. [14-17]). In 2011, Zhong proved a half-discrete Hilbert-type inequality with the non-homogeneous kernel as follows (cf. [18]): If $p>1, \frac{1}{p}+\frac{1}{q}=1,0<\lambda \leq 2, a_{n}, f(x) \geq 0, f(x)$ is a measurable function in $(0, \infty)$, such that $0<\sum_{n=1}^{\infty} n^{p\left(1-\frac{\lambda}{2}\right)-1} a_{n}^{p}<\infty$ and $0<\int_{0}^{\infty} x^{q\left(1-\frac{\lambda}{2}\right)-1} f^{q}(x) d x<\infty$, then

$$
\begin{aligned}
& \sum_{n=1}^{\infty} \int_{0}^{\infty} \frac{\ln (n x)}{(n x)^{\lambda}-1} f(x) a_{n} d x \\
& \quad<\left(\frac{\pi}{\lambda}\right)^{2}\left\{\sum_{n=1}^{\infty} n^{p\left(1-\frac{\lambda}{2}\right)-1} a_{n}^{p}\right\}^{\frac{1}{p}}\left\{\int_{0}^{\infty} x^{q\left(1-\frac{\lambda}{2}\right)-1} f^{q}(x) d x\right\}^{\frac{1}{q}},
\end{aligned}
$$

where the constant factor $\left(\frac{\pi}{\lambda}\right)^{2}$ is the best possible.

In this article, using the way of weight functions and the idea of introducing parameters, and by means of Hadamard's inequality, we give a more accurate reverse inequality of (4) with a best constant factor as follows: For $p<0, \frac{1}{p}+\frac{1}{q}=1$, we have

$$
\begin{aligned}
& \sum_{n=1}^{\infty} a_{n} \int_{0}^{\infty} \frac{\ln \left[x\left(n-\frac{1}{2}\right)\right]}{x^{\lambda}\left(n-\frac{1}{2}\right)^{\lambda}-1} f(x) d x \\
& \quad>\left(\frac{\pi}{\lambda}\right)^{2}\left\{\int_{0}^{\infty} x^{p\left(1-\frac{\lambda}{2}\right)-1} f^{p}(x) d x\right\}^{1 / p}\left\{\sum_{n=1}^{\infty}\left(n-\frac{1}{2}\right)^{q\left(1-\frac{\lambda}{2}\right)-1} a_{n}^{q}\right\}^{1 / q} .
\end{aligned}
$$

The main objective of this paper is to consider its best extension with parameters, the equivalent forms, as well as some particular cases.

\section{Some lemmas}

Lemma 1 If $0<\lambda_{1}<1, \lambda_{1}+\lambda_{2}=1$, then we have the following expression for the Beta function (cf. [1]):

$$
\int_{0}^{\infty} \frac{\ln u}{u-1} u^{\lambda_{1}-1} d u=\left[B\left(\lambda_{1}, \lambda_{2}\right)\right]^{2}=\left[\frac{\pi}{\sin \left(\pi \lambda_{1}\right)}\right]^{2}
$$

Lemma 2 Suppose that $\lambda>0,0<\sigma<\lambda \leq 1, \beta_{1} \in(-\infty, \infty), 0 \leq \beta_{2} \leq \frac{1}{2}, \delta \in\{-1,1\}$. We define the weight functions $\omega_{\sigma}(n)$ and $\widetilde{\omega}_{\sigma}(x)$ as follows:

$$
\begin{aligned}
& \omega_{\sigma}(n):=\left(n-\beta_{2}\right)^{\sigma} \int_{\beta_{1}}^{\infty} \frac{\left(x-\beta_{1}\right)^{\delta \sigma-1} \ln \left[\left(x-\beta_{1}\right)^{\delta}\left(n-\beta_{2}\right)\right]}{\left[\left(x-\beta_{1}\right)^{\delta}\left(n-\beta_{2}\right)\right]^{\lambda}-1} d x \quad(n \in \mathbf{N}), \\
& \widetilde{\omega}_{\sigma}(x):=\left(x-\beta_{1}\right)^{\delta \sigma} \sum_{n=1}^{\infty} \frac{\left(n-\beta_{2}\right)^{\sigma-1} \ln \left[\left(x-\beta_{1}\right)^{\delta}\left(n-\beta_{2}\right)\right]}{\left[\left(x-\beta_{1}\right)^{\delta}\left(n-\beta_{2}\right)\right]^{\lambda}-1} \quad\left(x \in\left(\beta_{1}, \infty\right)\right) .
\end{aligned}
$$


Setting $k_{\lambda}(\sigma):=\frac{1}{\lambda^{2}}\left[B\left(\frac{\sigma}{\lambda}, 1-\frac{\sigma}{\lambda}\right)\right]^{2}=\left[\frac{\pi}{\lambda \sin \left(\frac{\pi \sigma}{\lambda}\right)}\right]^{2}$, we have the following inequalities:

$$
\begin{aligned}
0 & <k_{\lambda}(\sigma)\left(1-\theta_{\lambda}(x)\right)<\widetilde{\omega}_{\sigma}(x)<\omega_{\sigma}(n)=k_{\lambda}(\sigma), \\
0 & <\theta_{\lambda}(x):=\frac{1}{\lambda^{2} k_{\lambda}(\sigma)} \int_{0}^{\left[\left(x-\beta_{1}\right)^{\delta}\left(1-\beta_{2}\right)\right]^{\lambda}} \frac{\ln v}{v-1} v^{\frac{\sigma}{\lambda}-1} d v \\
& =O\left(\left(x-\beta_{1}\right)^{\frac{\delta \sigma}{2}}\right) \quad\left(x \in\left(\beta_{1}, \infty\right)\right) .
\end{aligned}
$$

Proof Putting $u=\left[\left(x-\beta_{1}\right)^{\delta}\left(n-\beta_{2}\right)\right]^{\lambda}$ in (7), by Lemma 1, we find

$$
\omega_{\sigma}(n)=\frac{1}{\lambda^{2}} \int_{0}^{\infty} \frac{\ln u}{u-1} u^{\frac{\sigma}{\lambda}-1} d u=k_{\lambda}(\sigma)
$$

For fixed $x \in\left(\beta_{1}, \infty\right)$, setting

$$
f(t):=\frac{\left(x-\beta_{1}\right)^{\delta \sigma} \ln \left[\left(x-\beta_{1}\right)^{\delta}\left(t-\beta_{2}\right)\right]}{\left[\left(x-\beta_{1}\right)^{\delta}\left(t-\beta_{2}\right)\right]^{\lambda}-1}\left(t-\beta_{2}\right)^{\sigma-1} \quad\left(t \in\left(\beta_{2}, \infty\right)\right)
$$

in view of $0<\sigma<\lambda \leq 1$, we find $\left[\frac{\ln u}{u^{\lambda}-1}\right]^{\prime}<0,\left[\frac{\ln u}{u^{\lambda}-1}\right]^{\prime \prime}>0(u>0)(c f$. [19], Example 2.2.1) and then $f^{\prime}(t)<0$, and $f^{\prime \prime}(t)>0$. By the following Hermite-Hadamard inequality (cf. [20]):

$$
f(n)<\int_{n-\frac{1}{2}}^{n+\frac{1}{2}} f(t) d t \quad(n \in \mathbf{N})
$$

and putting $v=\left[\left(x-\beta_{1}\right)^{\delta}\left(t-\beta_{2}\right)\right]^{\lambda}$, it follows that

$$
\begin{aligned}
\tilde{\omega}_{\sigma}(x) & =\sum_{n=1}^{\infty} f(n)<\sum_{n=1}^{\infty} \int_{n-\frac{1}{2}}^{n+\frac{1}{2}} f(t) d t=\int_{\frac{1}{2}}^{\infty} f(t) d t \\
& \leq \int_{\beta_{2}}^{\infty} f(t) d t=\frac{1}{\lambda^{2}} \int_{0}^{\infty} \frac{\ln v}{v-1} v^{\frac{\sigma}{\lambda}-1} d v=k_{\lambda}(\sigma), \\
\tilde{\omega}_{\sigma}(x) & =\sum_{n=1}^{\infty} f(n)>\int_{1}^{\infty} f(t) d t=\int_{\beta_{2}}^{\infty} f(t) d t-\int_{\beta_{2}}^{1} f(t) d t \\
& =k_{\lambda}(\sigma)-\frac{1}{\lambda^{2}} \int_{0}^{\left[\left(x-\beta_{1}\right)^{\delta}\left(1-\beta_{2}\right)\right]^{\lambda}} \frac{\ln v}{v-1} v^{\frac{\sigma}{\lambda}-1} d v \\
& =k_{\lambda}(\sigma)\left(1-\theta_{\lambda}(x)\right)>0,
\end{aligned}
$$

where

$$
0<\theta_{\lambda}(x):=\frac{1}{\lambda^{2} k_{\lambda}(\sigma)} \int_{0}^{\left[\left(x-\beta_{1}\right)^{\delta}\left(1-\beta_{2}\right)\right]^{\lambda}} \frac{\ln v}{v-1} v^{\frac{\sigma}{\lambda}-1} d v \quad\left(x \in\left(\beta_{1}, \infty\right)\right)
$$

Since $\lim _{v \rightarrow 0^{+}} \frac{\ln v}{v-1} v^{\frac{\sigma}{2 \lambda}}=\lim _{v \rightarrow \infty} \frac{\ln v}{v-1} v^{\frac{\sigma}{2 \lambda}}=0$ and $\left.\frac{\ln v}{v-1} v^{\frac{\sigma}{2 \lambda}}\right|_{v=1}=1$, in view of the bounded properties of continuous function, there exists a constant $M>0$, such that $0<\frac{\ln v}{v-1} v^{\frac{\sigma}{2 \lambda}} \leq M$ 
$(v \in(0, \infty))$. For $x \in\left(\beta_{1}, \infty\right)$, we have

$$
\begin{aligned}
0 & <\int_{0}^{\left[\left(x-\beta_{1}\right)^{\delta}\left(1-\beta_{2}\right)\right]^{\lambda}} \frac{\ln v}{v-1} v^{\frac{\sigma}{\lambda}-1} d v \\
& =\int_{0}^{\left[\left(x-\beta_{1}\right)^{\delta}\left(1-\beta_{2}\right)\right]^{\lambda}}\left(\frac{\ln v}{v-1} v^{\frac{\sigma}{2 \lambda}}\right) v^{\frac{\sigma}{2 \lambda}-1} d v \\
& \leq M \int_{0}^{\left[\left(x-\beta_{1}\right)^{\delta}\left(1-\beta_{2}\right)\right]^{\lambda}} v^{\frac{\sigma}{2 \lambda}-1} d v \\
& =\frac{2 M \lambda}{\sigma}\left(1-\beta_{2}\right)^{\frac{\sigma}{2}}\left(x-\beta_{1}\right)^{\frac{\delta \sigma}{2}}
\end{aligned}
$$

Hence we proved that (9) and (10) are valid.

Lemma 3 Suppose that $\frac{1}{p}+\frac{1}{q}=1(p \neq 0,1), 0<\sigma<\lambda \leq 1, \beta_{1} \in(-\infty, \infty), 0 \leq \beta_{2} \leq \frac{1}{2}$, $\delta \in\{-1,1\}, a_{n} \geq 0, f(x)$ is a non-negative real measurable function in $\left(\beta_{1}, \infty\right)$. Then

(i) for $p>1$, we have the following inequalities:

$$
\begin{aligned}
J & :=\left\{\sum_{n=1}^{\infty}\left(n-\beta_{2}\right)^{p \sigma-1}\left[\int_{\beta_{1}}^{\infty} \frac{\ln \left[\left(x-\beta_{1}\right)^{\delta}\left(n-\beta_{2}\right)\right]}{\left[\left(x-\beta_{1}\right)^{\delta}\left(n-\beta_{2}\right)\right]^{\lambda}-1} f(x) d x\right]^{p}\right\}^{\frac{1}{p}} \\
& \leq\left[k_{\lambda}(\sigma)\right]^{\frac{1}{q}}\left\{\int_{\beta_{1}}^{\infty} \widetilde{\omega}_{\sigma}(x)\left(x-\beta_{1}\right)^{p(1-\delta \sigma)-1} f^{p}(x) d x\right\}^{\frac{1}{p}}, \\
L_{1} & :=\left\{\int_{\beta_{1}}^{\infty} \frac{\left(x-\beta_{1}\right)^{q \delta \sigma-1}}{\widetilde{\omega}_{\sigma}^{q-1}(x)}\left[\sum_{n=1}^{\infty} \frac{\ln \left[\left(x-\beta_{1}\right)^{\delta}\left(n-\beta_{2}\right)\right]}{\left[\left(x-\beta_{1}\right)^{\delta}\left(n-\beta_{2}\right)\right]^{\lambda}-1} a_{n}\right]^{q} d x\right\}^{\frac{1}{q}} \\
& \leq\left\{k_{\lambda}(\sigma) \sum_{n=1}^{\infty}\left(n-\beta_{2}\right)^{q(1-\sigma)-1} a_{n}^{q}\right\}^{\frac{1}{q}},
\end{aligned}
$$

where $\omega_{\sigma}(n)$ and $\widetilde{\omega}_{\sigma}(x)$ are defined by (7) and (8);

(ii) for $0<p<1$ or $p<0$, we have the reverses of (15) and (16).

Proof (i) By (7)-(10) and the Hölder inequality [20], we have

$$
\begin{aligned}
& {\left[\int_{\beta_{1}}^{\infty} \frac{\ln \left[\left(x-\beta_{1}\right)^{\delta}\left(n-\beta_{2}\right)\right]}{\left[\left(x-\beta_{1}\right)^{\delta}\left(n-\beta_{2}\right)\right]^{\lambda}-1} f(x) d x\right]^{p}} \\
& \quad=\left\{\int _ { \beta _ { 1 } } ^ { \infty } \frac { \operatorname { l n } [ ( x - \beta _ { 1 } ) ^ { \delta } ( n - \beta _ { 2 } ) ] } { [ ( x - \beta _ { 1 } ) ^ { \delta } ( n - \beta _ { 2 } ) ] ^ { \lambda } - 1 } \left[\frac{\left(x-\beta_{1}\right)^{(1-\delta \sigma) / q}}{\left.\left.\left(n-\beta_{2}\right)^{(1-\sigma) / p} f(x)\right]\left[\frac{\left(n-\beta_{2}\right)^{(1-\sigma) / p}}{\left(x-\beta_{1}\right)^{(1-\delta \sigma) / q}}\right] d x\right\}^{p}}\right.\right. \\
& \quad \leq \int_{\beta_{1}}^{\infty} \frac{\ln \left[\left(x-\beta_{1}\right)^{\delta}\left(n-\beta_{2}\right)\right]}{\left[\left(x-\beta_{1}\right)^{\delta}\left(n-\beta_{2}\right)\right]^{\lambda}-1} \frac{\left(x-\beta_{1}\right)^{(1-\delta \sigma)(p-1)}}{\left(n-\beta_{2}\right)^{1-\sigma}} f^{p}(x) d x \\
& \quad \times\left[\int_{\beta_{1}}^{\infty} \frac{\ln \left[\left(x-\beta_{1}\right)^{\delta}\left(n-\beta_{2}\right)\right]}{\left[\left(x-\beta_{1}\right)^{\delta}\left(n-\beta_{2}\right)\right]^{\lambda}-1} \frac{\left(n-\beta_{2}\right)^{(1-\sigma)(q-1)}}{\left(x-\beta_{1}\right)^{1-\delta \sigma}} d x\right]^{p-1} \\
& =\int_{\beta_{1}}^{\infty} \frac{f^{p}(x) \ln \left[\left(x-\beta_{1}\right)^{\delta}\left(n-\beta_{2}\right)\right]}{\left[\left(x-\beta_{1}\right)^{\delta}\left(n-\beta_{2}\right)\right]^{\lambda}-1} \frac{\left(x-\beta_{1}\right)^{(1-\delta \sigma)(p-1)}}{\left(n-\beta_{2}\right)^{1-\sigma}} d x\left[\left(n-\beta_{2}\right)^{q(1-\sigma)-1} \omega_{\sigma}(n)\right]^{p-1} \\
& =\frac{k_{\lambda}^{p-1}(\sigma)}{\left(n-\beta_{2}\right)^{p \sigma-1}} \int_{\beta_{1}}^{\infty} \frac{f^{p}(x) \ln \left[\left(x-\beta_{1}\right)^{\delta}\left(n-\beta_{2}\right)\right]}{\left[\left(x-\beta_{1}\right)^{\delta}\left(n-\beta_{2}\right)\right]^{\lambda}-1} \frac{\left(x-\beta_{1}\right)^{(1-\delta \sigma)(p-1)}}{\left(n-\beta_{2}\right)^{1-\sigma}} d x .
\end{aligned}
$$


By the Lebesgue term by term integration theorem [21], (17), and (9), we obtain

$$
\begin{aligned}
J^{p} & \leq k_{\lambda}^{p-1}(\sigma) \sum_{n=1}^{\infty} \int_{\beta_{1}}^{\infty} \frac{f^{p}(x) \ln \left[\left(x-\beta_{1}\right)^{\delta}\left(n-\beta_{2}\right)\right]}{\left[\left(x-\beta_{1}\right)^{\delta}\left(n-\beta_{2}\right)\right]^{\lambda}-1} \frac{\left(x-\beta_{1}\right)^{(1-\delta \sigma)(p-1)}}{\left(n-\beta_{2}\right)^{1-\sigma}} d x \\
& =k_{\lambda}^{p-1}(\sigma) \int_{\beta_{1}}^{\infty} \sum_{n=1}^{\infty} \frac{\ln \left[\left(x-\beta_{1}\right)^{\delta}\left(n-\beta_{2}\right)\right]}{\left[\left(x-\beta_{1}\right)^{\delta}\left(n-\beta_{2}\right)\right]^{\lambda}-1} \frac{\left(x-\beta_{1}\right)^{(1-\delta \sigma)(p-1)}}{\left(n-\beta_{2}\right)^{1-\sigma}} f^{p}(x) d x \\
& =k_{\lambda}^{p-1}(\sigma) \int_{\beta_{1}}^{\infty} \widetilde{\omega}_{\sigma}(x)\left(x-\beta_{1}\right)^{p(1-\delta \sigma)-1} f^{p}(x) d x
\end{aligned}
$$

Hence (15) is valid. Using the Hölder inequality, in view of the Lebesgue term by term integration theorem and (9) again, we have

$$
\begin{aligned}
& \quad\left[\sum_{n=1}^{\infty} \frac{\ln \left[\left(x-\beta_{1}\right)^{\delta}\left(n-\beta_{2}\right)\right]}{\left[\left(x-\beta_{1}\right)^{\delta}\left(n-\beta_{2}\right)\right]^{\lambda}-1} a_{n}\right]^{q} \\
& \quad=\left\{\sum_{n=1}^{\infty} \frac{\ln \left[\left(x-\beta_{1}\right)^{\delta}\left(n-\beta_{2}\right)\right]}{\left[\left(x-\beta_{1} \delta^{\delta}\left(n-\beta_{2}\right)\right]^{\lambda}-1\right.} \frac{\left(x-\beta_{1}\right)^{(1-\delta \sigma) / q}}{\left(n-\beta_{2}\right)^{(1-\sigma) / p}} \frac{\left(n-\beta_{2}\right)^{(1-\sigma) / p} a_{n}}{\left(x-\beta_{1}\right)^{(1-\delta \sigma) / q}}\right\} \\
& \quad \leq\left[\widetilde{\omega}_{\sigma}(x)\left(x-\beta_{1}\right)^{p(1-\delta \sigma)-1}\right]^{q-1} \sum_{n=1}^{\infty} \frac{\ln \left[\left(x-\beta_{1}\right)^{\delta}\left(n-\beta_{2}\right)\right]}{\left[\left(x-\beta_{1}\right)^{\delta}\left(n-\beta_{2}\right)\right]^{\lambda}-1} \frac{\left(n-\beta_{2}\right)^{(1-\sigma)(q-1)} a_{n}^{q}}{\left(x-\beta_{1}\right)^{1-\delta \sigma}} \\
& \quad=\widetilde{\omega}_{\sigma}^{q-1}(x)\left(x-\beta_{1}\right)^{1-q \delta \sigma} \sum_{n=1}^{\infty} \frac{\ln \left[\left(x-\beta_{1}\right)^{\delta}\left(n-\beta_{2}\right)\right]}{\left[\left(x-\beta_{1}\right)^{\delta}\left(n-\beta_{2}\right)\right]^{\lambda}-1} \frac{\left(n-\beta_{2}\right)^{(1-\sigma)(q-1)}}{\left(x-\beta_{1}\right)^{1-\delta \sigma}} a_{n}^{q},
\end{aligned}
$$

and therefore

$$
\begin{aligned}
L_{1}^{q} & \leq \int_{\beta_{1}}^{\infty} \sum_{n=1}^{\infty} \frac{\ln \left[\left(x-\beta_{1}\right)^{\delta}\left(n-\beta_{2}\right)\right]}{\left[\left(x-\beta_{1}\right)^{\delta}\left(n-\beta_{2}\right)\right]^{\lambda}-1} \frac{\left(n-\beta_{2}\right)^{(1-\delta \sigma)(q-1)}}{\left(x-\beta_{1}\right)^{1-\delta \sigma}} a_{n}^{q} d x \\
& =\sum_{n=1}^{\infty}\left[\left(n-\beta_{2}\right)^{\sigma} \int_{\beta_{1}}^{\infty} \frac{\left(x-\beta_{1}\right)^{\delta \sigma-1} \ln \left[\left(x-\beta_{1}\right)^{\delta}\left(n-\beta_{2}\right)\right]}{\left[\left(x-\beta_{1}\right)^{\delta}\left(n-\beta_{2}\right)\right]^{\lambda}-1} d x\right]\left(n-\beta_{2}\right)^{q(1-\sigma)-1} a_{n}^{q} \\
& =\sum_{n=1}^{\infty} \omega_{\sigma}(n)\left(n-\beta_{2}\right)^{q(1-\sigma)-1} a_{n}^{q}=k_{\lambda}(\sigma) \sum_{n=1}^{\infty}\left(n-\beta_{2}\right)^{q(1-\sigma)-1} a_{n}^{q} .
\end{aligned}
$$

Hence (16) is valid.

(ii) For $0<p<1(q<0)$ or $p<0(0<q<1)$, using the reverse Hölder inequality ( $c f$. [20]) and in the same way, we obtain the reverses of (15) and (16).

Definition 1 As the assumptions of Lemma 2 and Lemma 3, we define $\phi(x):=(x-$ $\left.\beta_{1}\right)^{p(1-\delta \sigma)-1}, \widetilde{\phi}(x):=\left(1-\theta_{\lambda}(x)\right) \phi(x), \psi(n):=\left(n-\beta_{2}\right)^{q(1-\sigma)-1}$, and the following sets:

$$
\begin{aligned}
& L_{p, \phi}\left(\beta_{1}, \infty\right):=\left\{f ;\|f\|_{p, \phi}=\left[\int_{\beta_{1}}^{\infty} \phi(x)|f(x)|^{p} d x\right]^{1 / p}<\infty\right\}, \\
& l_{q, \psi}:=\left\{a=\left\{a_{n}\right\} ;\|a\|_{q, \psi}=\left[\sum_{n=1}^{\infty} \psi(n)\left|a_{n}\right|^{q}\right]^{1 / q}<\infty\right\} .
\end{aligned}
$$


Note If $p>1$, then $L_{p, \phi}\left(\beta_{1}, \infty\right)$ and $l_{q, \psi}$ are normed spaces; if $0<p<1$ or $p<0$, then both $L_{p, \phi}\left(\beta_{1}, \infty\right)$ and $l_{q, \psi}$ are not normed spaces, but we still use the formal symbols in the following.

\section{Main results and applications}

Theorem 1 Suppose that $p<0, \frac{1}{p}+\frac{1}{q}=1,0<\sigma<\lambda \leq 1, \beta_{1} \in(-\infty,+\infty), 0 \leq \beta_{2} \leq \frac{1}{2}$, $\delta \in\{-1,1\}, f(x), a_{n} \geq 0$, satisfying $f \in L_{p, \phi}\left(\beta_{1}, \infty\right), a=\left\{a_{n}\right\}_{n=1}^{\infty} \in l_{q, \psi},\|f\|_{p, \phi}>0,\|a\|_{q, \psi}>0$. Then we have the following equivalent inequalities:

$$
\begin{aligned}
I & :=\sum_{n=1}^{\infty} a_{n} \int_{\beta_{1}}^{\infty} \frac{\ln \left[\left(x-\beta_{1}\right)^{\delta}\left(n-\beta_{2}\right)\right]}{\left[\left(x-\beta_{1}\right)^{\delta}\left(n-\beta_{2}\right)\right]^{\lambda}-1} f(x) d x \\
& =\int_{\beta_{1}}^{\infty} f(x) \sum_{n=1}^{\infty} \frac{\ln \left[\left(x-\beta_{1}\right)^{\delta}\left(n-\beta_{2}\right)\right] a_{n}}{\left[\left(x-\beta_{1}\right)^{\delta}\left(n-\beta_{2}\right)\right]^{\lambda}-1} d x>k_{\lambda}(\sigma)\|f\|_{p, \phi}\|a\|_{q, \psi}, \\
J & =\left\{\sum_{n=1}^{\infty}\left(n-\beta_{2}\right)^{p \sigma-1}\left[\int_{\beta_{1}}^{\infty} \frac{\ln \left[\left(x-\beta_{1}\right)^{\delta}\left(n-\beta_{2}\right)\right] f(x)}{\left[\left(x-\beta_{1}\right)^{\delta}\left(n-\beta_{2}\right)\right]^{\lambda}-1} d x\right]^{p}\right\}^{\frac{1}{p}}>k_{\lambda}(\sigma)\|f\|_{p, \phi}, \\
L & :=\left\{\int_{\beta_{1}}^{\infty}\left(x-\beta_{1}\right)^{q \delta \sigma-1}\left[\sum_{n=1}^{\infty} \frac{\ln \left[\left(x-\beta_{1}\right)^{\delta}\left(n-\beta_{2}\right)\right] a_{n}}{\left[\left(x-\beta_{1}\right)^{\delta}\left(n-\beta_{2}\right)\right]^{\lambda}-1}\right]^{q} d x\right\}^{\frac{1}{q}}>k_{\lambda}(\sigma)\|a\|_{q, \psi},
\end{aligned}
$$

where the constant factor $k_{\lambda}(\sigma)=\left[\frac{\pi}{\lambda \sin \left(\frac{\pi \sigma}{\lambda}\right)}\right]^{2}$ is the best possible.

Proof By the Lebesgue term by term integration theorem [21], we find that there are two expressions of $I$ in (21). By (9), the reverse of (15) and $0<\|f\|_{p, \phi}<\infty$, we have (22). By the reverse Hölder inequality, we find

$$
\begin{aligned}
I & =\sum_{n=1}^{\infty}\left[\left(n-\beta_{2}\right)^{\sigma-\frac{1}{p}} \int_{\beta_{1}}^{\infty} \frac{\ln \left[\left(x-\beta_{1}\right)^{\delta}\left(n-\beta_{2}\right)\right] f(x)}{\left[\left(x-\beta_{1}\right)^{\delta}\left(n-\beta_{2}\right)\right]^{\lambda}-1} d x\right]\left[\left(n-\beta_{2}\right)^{\frac{1}{p}-\sigma} a_{n}\right] \\
& \geq J\left\{\sum_{n=1}^{\infty}\left[\left(n-\beta_{2}\right)^{q(1-\sigma)-1} a_{n}^{q}\right\}^{1 / q}=J\|a\|_{q, \psi} .\right.
\end{aligned}
$$

Then by (22), (21) is valid. On the other hand, assuming that (21) is valid, setting

$$
a_{n}:=\left(n-\beta_{2}\right)^{p \sigma-1}\left[\int_{\beta_{1}}^{\infty} \frac{\ln \left[\left(x-\beta_{1}\right)^{\delta}\left(n-\beta_{2}\right)\right]}{\left[\left(x-\beta_{1}\right)^{\delta}\left(n-\beta_{2}\right)\right]^{\lambda}-1} f(x) d x\right]^{p-1} \quad(n \in \mathbf{N}),
$$

then by (21), we have

$$
\|a\|_{q, \psi}^{q}=\sum_{n=1}^{\infty}\left(n-\beta_{2}\right)^{q(1-\sigma)-1} a_{n}^{q}=J^{p}=I \geq k_{\lambda}(\sigma)\|f\|_{p, \phi}\|a\|_{q, \psi} .
$$

By (9), the reverse of (15), and $0<\|f\|_{p, \phi}<\infty$, it follows that $J>0$. If $J=\infty$, then (22) is trivially valid; if $J<\infty$, then $0<\|a\|_{q, \psi}=J^{p-1}<\infty$. Thus the conditions of applying (21) are fulfilled and by (21), (26) takes a strict sign inequality. Thus we find

$$
J=\|a\|_{q, \psi}^{q-1}>k_{\lambda}(\sigma)\|f\|_{p, \phi}
$$

Hence, (22) is valid, which is equivalent to (21). 
By (9), the reverse of (16) and $0<\|a\|_{q, \psi}<\infty$, we obtain (23). By the reverse Hölder inequality again, we have

$$
\begin{aligned}
I & =\int_{\beta_{1}}^{\infty}\left[\left(x-\beta_{1}\right)^{\delta \sigma-\frac{1}{q}} \sum_{n=1}^{\infty} \frac{\ln \left[\left(x-\beta_{1}\right)^{\delta}\left(n-\beta_{2}\right)\right] a_{n}}{\left[\left(x-\beta_{1}\right)^{\delta}\left(n-\beta_{2}\right)\right]^{\lambda}-1}\right]\left[\left(x-\beta_{1}\right)^{\frac{1}{q}-\delta \sigma} f(x)\right] d x \\
& \geq L\left\{\int_{\beta_{1}}^{\infty}\left(x-\beta_{1}\right)^{p(1-\delta \sigma)-1} f^{p}(x) d x\right\}^{1 / p}=L\|f\|_{p, \phi} .
\end{aligned}
$$

Hence (21) is valid by using (23). On the other hand, assuming that (21) is valid, setting

$$
f(x):=\left(x-\beta_{1}\right)^{q \delta \sigma-1}\left[\sum_{n=1}^{\infty} \frac{\ln \left[\left(x-\beta_{1}\right)^{\delta}\left(n-\beta_{2}\right)\right] a_{n}}{\left[\left(x-\beta_{1}\right)^{\delta}\left(n-\beta_{2}\right)\right]^{\lambda}-1}\right]^{q-1} \quad\left(x \in\left(\beta_{1}, \infty\right)\right),
$$

then by (21), we find

$$
\|f\|_{p, \phi}^{p}=\int_{\beta_{1}}^{\infty}\left(x-\beta_{1}\right)^{p(1-\delta \sigma)-1} f^{p}(x) d x=L^{q}=I \geq k_{\lambda}(\sigma)\|f\|_{p, \phi}\|a\|_{q, \psi} .
$$

By (9), the reverse of (16) and $0<\|a\|_{q, \psi}<\infty$, it follows that $L>0$. If $L=\infty$, then (23) is trivially valid; if $L<\infty$, then $0<\|f\|_{p, \phi}=L^{q-1}<\infty$, i.e. the conditions of applying (21) are fulfilled and by (30), we still have

$$
\|f\|_{p, \phi}^{p}=L^{q}=I>k_{\lambda}(\sigma)\|f\|_{p, \phi}\|a\|_{q, \psi} \text {, i.e. } L=\|f\|_{p, \phi}^{p-1}>k_{\lambda}(\sigma)\|a\|_{q, \psi} \text {. }
$$

Hence (23) is valid, which is equivalent to (21).

It follows that (21), (22), and (23) are equivalent.

We prove that the constant factor in (21) is the best possible. For $0<\varepsilon<q \sigma$, we set $E_{\delta}:=\left\{x \mid 0<\left(x-\beta_{1}\right)^{\delta}<1\right\}, \widetilde{a}=\left\{\widetilde{a}_{n}\right\}_{n=1}^{\infty}$, and $\widetilde{f}(x)$ as follows:

$$
\widetilde{a}_{n}=\left(n-\beta_{2}\right)^{\sigma-\frac{\varepsilon}{q}-1} ; \quad \tilde{f}(x)= \begin{cases}\left(x-\beta_{1}\right)^{\delta\left(\sigma+\frac{\varepsilon}{p}\right)-1}, & x \in E_{\delta}, \\ 0, & x \in\left(\beta_{1}, \infty\right) \backslash E_{\delta} .\end{cases}
$$

If there exists a positive number $k \geq k_{\lambda}(\sigma)$, such that (21) is still valid as we replace $k_{\lambda}(\sigma)$ by $k$, then in particular, on substitution of $\widetilde{a}$ and $\widetilde{f}(x)$, we have

$$
\widetilde{I}:=\sum_{n=1}^{\infty} \widetilde{a}_{n} \int_{\beta_{1}}^{\infty} \frac{\ln \left[\left(x-\beta_{1}\right)^{\delta}\left(n-\beta_{2}\right)\right]}{\left[\left(x-\beta_{1}\right)^{\delta}\left(n-\beta_{2}\right)\right]^{\lambda}-1} \widetilde{f}(x) d x>k\|\widetilde{f}\|_{p, \phi}\|\widetilde{a}\|_{q, \psi} .
$$

For $p<0,0<q<1$, setting $\widetilde{\sigma}=\sigma-\frac{\varepsilon}{q}$, we find by Lemma 2 that

$$
\begin{aligned}
\widetilde{I} & =\int_{E_{\delta}}\left(x-\beta_{1}\right)^{\delta \varepsilon-1}\left(x-\beta_{1}\right)^{\delta\left(\sigma-\frac{\varepsilon}{q}\right)} \sum_{n=1}^{\infty} \frac{\ln \left[\left(x-\beta_{1}\right)^{\delta}\left(n-\beta_{2}\right)\right]}{\left[\left(x-\beta_{1}\right)^{\delta}\left(n-\beta_{2}\right)\right]^{\lambda}-1}\left(n-\beta_{2}\right)^{\left(\sigma-\frac{\varepsilon}{q}\right)-1} d x \\
& =\int_{E_{\delta}}\left(x-\beta_{1}\right)^{\delta \varepsilon-1} \widetilde{\omega}_{\widetilde{\sigma}}(x) d x<k_{\lambda}(\widetilde{\sigma}) \int_{E_{\delta}}\left(x-\beta_{1}\right)^{\delta \varepsilon-1} d x \\
& =\frac{1}{\varepsilon} k_{\lambda}\left(\sigma-\frac{\varepsilon}{q}\right) .
\end{aligned}
$$


Setting $u=\left(x-\beta_{1}\right)^{\delta}$, by calculation we obtain

$$
\begin{aligned}
\|\widetilde{f}\|_{p, \phi} & =\left\{\int_{\beta_{1}}^{\infty}\left(x-\beta_{1}\right)^{p(1-\delta \sigma)-1} \widetilde{f}^{p}(x) d x\right\}^{1 / p} \\
& =\left\{\int_{E_{\delta}}\left(x-\beta_{1}\right)^{-1+\delta \varepsilon} d x\right\}^{1 / p}=\left\{\int_{0}^{1} u^{-1+\varepsilon} d u\right\}^{1 / p}=\left(\frac{1}{\varepsilon}\right)^{1 / p}, \\
\|\widetilde{a}\|_{q, \psi}^{q} & =\sum_{n=1}^{\infty}\left(n-\beta_{2}\right)^{q(1-\sigma)-1} \widetilde{a}_{n}^{q}=\sum_{n=1}^{\infty}\left(n-\beta_{2}\right)^{-1-\varepsilon} \\
& >\int_{1}^{\infty}\left(x-\beta_{2}\right)^{-1-\varepsilon} d x=\frac{1}{\varepsilon\left(1-\beta_{2}\right)^{\varepsilon}} .
\end{aligned}
$$

In view of (32), (33) and $0<q<1$, it follows that

$$
k_{\lambda}\left(\sigma-\frac{\varepsilon}{q}\right)>\varepsilon \widetilde{I}>\varepsilon k\|\widetilde{f}\|_{p, \phi}\|\widetilde{a}\|_{q, \psi}>k\left[\frac{1}{\left(1-\beta_{2}\right)^{\varepsilon}}\right]^{1 / q} .
$$

For $\varepsilon \rightarrow 0^{+}$in (36), we have $k_{\lambda}(\sigma) \geq k$. Hence $k=k_{\lambda}(\sigma)$ is the best value of (21). We confirm that the constant factor $k_{\lambda}(\sigma)$ in (22) ((23)) is the best possible. Otherwise we can get the contradiction by (24) ((28)) that the constant factor in (21) is not the best possible.

Theorem 2 Suppose that $0<p<1, \frac{1}{p}+\frac{1}{q}=1,0<\sigma<\lambda \leq 1, \beta_{1} \in(-\infty,+\infty), 0 \leq \beta_{2} \leq \frac{1}{2}$, $\delta \in\{-1,1\}, f(x), a_{n} \geq 0$, satisfying $f \in L_{p, \widetilde{\phi}}\left(\beta_{1}, \infty\right), a=\left\{a_{n}\right\}_{n=1}^{\infty} \in l_{q, \psi},\|f\|_{p, \widetilde{\phi}}>0,\|a\|_{q, \psi}>0$.

Then we have the following equivalent inequalities:

$$
\begin{aligned}
I & =\sum_{n=1}^{\infty} a_{n} \int_{\beta_{1}}^{\infty} \frac{\ln \left[\left(x-\beta_{1}\right)^{\delta}\left(n-\beta_{2}\right)\right]}{\left[\left(x-\beta_{1}\right)^{\delta}\left(n-\beta_{2}\right)\right]^{\lambda}-1} f(x) d x \\
& =\int_{\beta_{1}}^{\infty} f(x) \sum_{n=1}^{\infty} \frac{\ln \left[\left(x-\beta_{1}\right)^{\delta}\left(n-\beta_{2}\right)\right] a_{n}}{\left[\left(x-\beta_{1}\right)^{\delta}\left(n-\beta_{2}\right)\right]^{\lambda}-1} d x>k_{\lambda}(\sigma)\|f\|_{p, \tilde{\phi}}\|a\|_{q, \psi}, \\
J & =\left\{\sum_{n=1}^{\infty}\left(n-\beta_{2}\right)^{p \sigma-1}\left[\int_{\beta_{1}}^{\infty} \frac{\ln \left[\left(x-\beta_{1}\right)^{\delta}\left(n-\beta_{2}\right)\right] f(x)}{\left[\left(x-\beta_{1}\right)^{\delta}\left(n-\beta_{2}\right)\right]^{\lambda}-1} d x\right]^{p}\right\}^{\frac{1}{p}}>k_{\lambda}(\sigma)\|f\|_{p, \tilde{\phi}}, \\
\widetilde{L} & :=\left\{\int_{\beta_{1}}^{\infty} \frac{\left(x-\beta_{1}\right)^{q \delta \sigma-1}}{\left[\left(1-\theta_{\lambda}(x)\right]^{q-1}\right.}\left[\sum_{n=1}^{\infty} \frac{\ln \left[\left(x-\beta_{1}\right)^{\delta}\left(n-\beta_{2}\right)\right] a_{n}}{\left[\left(x-\beta_{1}\right)^{\delta}\left(n-\beta_{2}\right)\right]^{\lambda}-1}\right]^{q} d x\right\}^{\frac{1}{q}} \\
& >k_{\lambda}(\sigma)\|a\|_{q, \psi},
\end{aligned}
$$

where the constant factor $k_{\lambda}(\sigma)=\left[\frac{\pi}{\lambda \sin \left(\frac{\pi \sigma}{\lambda}\right)}\right]^{2}$ is the best possible.

Proof By (9), the reverse of (15) and $0<\|f\|_{p, \tilde{\phi}}<\infty$, we have (38). Using the reverse Hölder inequality, we obtain the reverse form of (24) as follows:

$$
I \geq J\|a\|_{q, \psi} .
$$

Then by (38), (37) is valid. 
On the other hand, if (37) is valid, setting $a_{n}$ as (25), then (26) still holds with $0<p<1$. By (37), we have

$$
\|a\|_{q, \psi}^{q}=\sum_{n=1}^{\infty}\left(n-\beta_{2}\right)^{q(1-\sigma)-1} a_{n}^{q}=J^{p}=I \geq k_{\lambda}(\sigma)\|f\|_{p, \tilde{\phi}}\|a\|_{q, \psi} .
$$

Then by (9), the reverse of (18) and $0<\|f\|_{p, \widetilde{\phi}}<\infty$, it follows that

$$
J=\left\{\sum_{n=1}^{\infty}\left(n-\beta_{2}\right)^{q(1-\sigma)-1} a_{n}^{q}\right\}^{1 / p}>0 .
$$

If $J=\infty$, then (38) is trivially valid; if $J<\infty$, then $0<\|a\|_{q, \psi}=J^{p-1}<\infty$, i.e. the conditions of applying (37) are fulfilled and by (41), we still have

$$
\|a\|_{q, \psi}^{q}=J^{p}=I>k_{\lambda}(\sigma)\|f\|_{p, \tilde{\phi}}\|a\|_{q, \psi}, \quad \text { i.e. } J=\|a\|_{q, \psi}^{q-1}>k_{\lambda}(\sigma)\|f\|_{p, \tilde{\phi}}
$$

Hence (38) is valid, which is equivalent to (37).

By the reverse of $(16)$, in view of $\widetilde{\omega}_{\sigma}(x)>k_{\lambda}(\sigma)\left(1-\theta_{\lambda}(x)\right)$ and $q<0$, we have

$$
\widetilde{L}>k_{\lambda}^{\frac{q-1}{q}}(\sigma) L_{1} \geq k_{\lambda}^{\frac{1}{p}}(\sigma)\left\{k_{\lambda}(\sigma) \sum_{n=1}^{\infty}\left(n-\beta_{2}\right)^{q(1-\sigma)-1} a_{n}^{q}\right\}^{\frac{1}{q}}=k_{\lambda}(\sigma)\|a\|_{q, \psi},
$$

then (39) is valid. By the reverse Hölder inequality again, we have

$$
\begin{aligned}
I= & \int_{\beta_{1}}^{\infty}\left[\frac{\left(x-\beta_{1}\right)^{\delta \sigma-\frac{1}{q}}}{\left(1-\theta_{\lambda}(x)\right)^{\frac{1}{p}}} \sum_{n=1}^{\infty} \frac{\ln \left[\left(x-\beta_{1}\right)^{\delta}\left(n-\beta_{2}\right)\right]}{\left[\left(x-\beta_{1}\right)^{\delta}\left(n-\beta_{2}\right)\right]^{\lambda}-1} a_{n}\right] \\
& \times\left[\left(1-\theta_{\lambda}(x)\right)^{\frac{1}{p}}\left(x-\beta_{1}\right)^{\frac{1}{q}-\delta \sigma} f(x)\right] d x \geq \widetilde{L}\|f\|_{p, \tilde{\phi}}
\end{aligned}
$$

Hence (37) is valid by (39). On the other hand, if (37) is valid, setting

$$
f(x)=\frac{\left(x-\beta_{1}\right)^{q \delta \sigma-1}}{\left[1-\theta_{\lambda}(x)\right]^{q-1}}\left[\sum_{n=1}^{\infty} \frac{\ln \left[\left(x-\beta_{1}\right)^{\delta}\left(n-\beta_{2}\right)\right] a_{n}}{\left[\left(x-\beta_{1}\right)^{\delta}\left(n-\beta_{2}\right)\right]^{\lambda}-1}\right]^{q-1} \quad\left(x \in\left(\beta_{1}, \infty\right)\right)
$$

then by the reverse of (16) and $0<\|a\|_{q, \psi}<\infty$, it follows that

$$
\widetilde{L}=\left\{\int_{\beta_{1}}^{\infty}\left[1-\theta_{\lambda}(x)\right]^{\frac{1}{p}}\left(x-\beta_{1}\right)^{p(1-\delta \sigma)-1} f^{p}(x) d x\right\}^{\frac{1}{q}}=\|f\|_{p, \widetilde{\phi}}^{p-1}>0 .
$$

If $\widetilde{L}=\infty$, then (39) is trivially valid; if $\widetilde{L}<\infty$, then $0<\|f\|_{p, \widetilde{\phi}}=\widetilde{L}^{q-1}<\infty$, i.e. the conditions of applying (37) are fulfilled and we have

$$
\|f\|_{p, \tilde{\phi}}^{p}=\widetilde{L}^{q}=I>k_{\lambda}(\sigma)\|f\|_{p, \widetilde{\phi}}\|a\|_{q, \psi}, \quad \text { i.e. } \widetilde{L}=\|f\|_{p, \tilde{\phi}}^{p-1}>k_{\lambda}(\sigma)\|a\|_{q, \psi} .
$$

Hence (39) is valid, which is equivalent to (37). It follows that (37), (38), and (39) are equivalent. 
If there exists a positive number $K \geq k_{\lambda}(\sigma)$, such that (37) is still valid as we replace $k_{\lambda}(\sigma)$ by $K$, then in particular, we have

$$
\widetilde{I}=\sum_{n=1}^{\infty} \widetilde{a}_{n} \int_{\beta_{1}}^{\infty} \frac{\ln \left[\left(x-\beta_{1}\right)^{\delta}\left(n-\beta_{2}\right)\right]}{\left[\left(x-\beta_{1}\right)^{\delta}\left(n-\beta_{2}\right)\right]^{\lambda}-1} \tilde{f}(x) d x>K\|\widetilde{f}\|_{p, \widetilde{\phi}}\|\widetilde{a}\|_{q, \psi},
$$

where $\widetilde{a}=\left\{\widetilde{a}_{n}\right\}_{n=1}^{\infty}$ and $\tilde{f}$ are taken as (31) $(0<\varepsilon<p(\lambda-\sigma))$. We find

$$
\begin{aligned}
\|\widetilde{f}\|_{p, \widetilde{\phi}} & =\left\{\int_{E_{\delta}}\left[1-O\left(\left(x-\beta_{1}\right)^{\frac{\delta \widetilde{\sigma}}{2}}\right)\right]\left(x-\beta_{1}\right)^{\delta \varepsilon-1} d x\right\}^{1 / p} \\
& =\left(\frac{1}{\varepsilon}-O(1)\right)^{1 / p} \cdot
\end{aligned}
$$

Since by (35) and setting $u=\left[\left(x-\beta_{1}\right)^{\delta}\left(n-\beta_{2}\right)\right]^{\lambda}$, it follows that

$$
\begin{aligned}
\widetilde{I} & =\sum_{n=1}^{\infty}\left(n-\beta_{2}\right)^{\sigma-\frac{\varepsilon}{q}-1} \int_{E_{\delta}} \frac{\ln \left[\left(x-\beta_{1}\right)^{\delta}\left(n-\beta_{2}\right)\right]}{\left[\left(x-\beta_{1}\right)^{\delta}\left(n-\beta_{2}\right)\right]^{\lambda}-1}\left(x-\beta_{1}\right)^{\delta\left(\sigma+\frac{\varepsilon}{p}\right)-1} d x \\
& \leq \sum_{n=1}^{\infty}\left(n-\beta_{2}\right)^{\sigma-\frac{\varepsilon}{q}-1} \int_{\beta_{1}}^{\infty} \frac{\ln \left[\left(x-\beta_{1}\right)^{\delta}\left(n-\beta_{2}\right)\right]}{\left[\left(x-\beta_{1}\right)^{\delta}\left(n-\beta_{2}\right)\right]^{\lambda}-1}\left(x-\beta_{1}\right)^{\delta\left(\sigma+\frac{\varepsilon}{p}\right)-1} d x \\
& =\frac{1}{\lambda^{2}} \sum_{n=1}^{\infty}\left(n-\beta_{2}\right)^{-\varepsilon-1} \int_{0}^{\infty} \frac{\ln u}{u-1} u^{\frac{\sigma}{\lambda}+\frac{\varepsilon}{p \lambda}-1} d u \\
& \leq\left[\frac{1}{\lambda} \sin \frac{\pi}{\lambda}\left(\sigma+\frac{\varepsilon}{p}\right)\right]^{2} \frac{\varepsilon+1-\beta_{2}}{\varepsilon\left(1-\beta_{2}\right)^{\varepsilon+1}},
\end{aligned}
$$

by (35), (43), and (44), we have (notice that $q<0$ )

$$
\begin{aligned}
& {\left[\frac{1}{\lambda} \sin \frac{\pi}{\lambda}\left(\sigma+\frac{\varepsilon}{p}\right)\right]^{2} \frac{\varepsilon+1-\beta_{2}}{\left(1-\beta_{2}\right)^{\varepsilon+1}}} \\
& \quad \geq \varepsilon \widetilde{I}>\varepsilon K\left(\frac{1}{\varepsilon}-O(1)\right)^{1 / p}\left(\frac{\varepsilon+1-\beta_{2}}{\varepsilon\left(1-\beta_{2}\right)^{\varepsilon+1}}\right)^{1 / q} \\
& \quad=K(1-\varepsilon O(1))^{1 / p}\left(\frac{\varepsilon+1-\beta_{2}}{\left(1-\beta_{2}\right)^{\varepsilon+1}}\right)^{1 / q} .
\end{aligned}
$$

For $\varepsilon \rightarrow 0^{+}$in (45), we obtain $k_{\lambda}(\sigma)=\left[\frac{1}{\lambda} \sin \left(\frac{\pi \sigma}{\lambda}\right)\right]^{2} \geq K$. Hence $k_{\lambda}(\sigma)=K$ is the best value of (37). We confirm that the constant factor $k_{\lambda}(\sigma)$ in (38) ((39)) is the best possible. Otherwise we can get the contradiction by $(40)((42))$ that the constant factor in (37) is not the best possible.

Remark 1 (i) For $\beta_{1}=\beta_{2}=0, \sigma=\frac{\lambda}{2}, \delta=1$ in (21), we have the reverse of (4). In particular, for $\lambda=1, p=q=2$ in the reverse of (4), we have

$$
\sum_{n=1}^{\infty} a_{n} \int_{0}^{\infty} \frac{\ln (n x)}{n x-1} f(x) d x>\pi^{2}\left\{\sum_{n=1}^{\infty} a_{n}^{2} \int_{0}^{\infty} f^{2}(x) d x\right\}^{\frac{1}{2}}
$$


(ii) For $\beta_{1}=0, \beta_{2}=\frac{1}{2}, \sigma=\frac{\lambda}{2}, \delta=1$ in (21), and (22), it follows from (5) that

$$
\sum_{n=1}^{\infty} x^{\frac{q \lambda}{2}-1}\left\{\int_{0}^{\infty} \frac{\ln \left[x\left(n-\frac{1}{2}\right)\right]}{\left[x\left(n-\frac{1}{2}\right)\right]^{\lambda}-1} a_{n}\right\}^{q} d x>\left(\frac{\pi}{\lambda}\right)^{2 q} \sum_{n=1}^{\infty}\left(n-\frac{1}{2}\right)^{q\left(1-\frac{\lambda}{2}\right)-1} a_{n}^{q} .
$$

In particular, for $\lambda=1, p=q=2$ in (5), we obtain

$$
\sum_{n=1}^{\infty} a_{n} \int_{0}^{\infty} \frac{\ln \left[x\left(n-\frac{1}{2}\right)\right]}{x\left(n-\frac{1}{2}\right)-1} f(x) d x>\pi^{2}\left(\sum_{n=1}^{\infty} a_{n}^{2} \int_{0}^{\infty} f^{2}(x) d x\right)^{1 / 2}
$$

which is a more accurate inequality than (46).

Remark 2 For $\delta=-1, \mu=\lambda-\sigma(>0)$ in Theorem 1, setting $\varphi(x):=\left(x-\beta_{1}\right)^{p(1-\mu)-1}$, and $F(x):=\left(x-\beta_{1}\right)^{\lambda} f(x)$, we have the following equivalent inequalities with the homogeneous kernel and the best possible constant factor $k_{\lambda}(\sigma)$ :

$$
\begin{aligned}
& \sum_{n=1}^{\infty} a_{n} \int_{\beta_{1}}^{\infty} \frac{\ln \left[\left(n-\beta_{2}\right) /\left(x-\beta_{1}\right)\right]}{\left(n-\beta_{2}\right)^{\lambda}-\left(x-\beta_{1}\right)^{\lambda}} F(x) d x \\
& \quad=\int_{\beta_{1}}^{\infty} F(x) \sum_{n=1}^{\infty} \frac{\ln \left[\left(n-\beta_{2}\right) /\left(x-\beta_{1}\right)\right]}{\left(n-\beta_{2}\right)^{\lambda}-\left(x-\beta_{1}\right)^{\lambda}} a_{n} d x>k_{\lambda}(\sigma)\|F\|_{p, \varphi}\|a\|_{q, \psi}, \\
& \left\{\sum_{n=1}^{\infty}\left(n-\beta_{2}\right)^{p \sigma-1}\left[\int_{\beta_{1}}^{\infty} \frac{\ln \left[\left(n-\beta_{2}\right) /\left(x-\beta_{1}\right)\right] F(x)}{\left(n-\beta_{2}\right)^{\lambda}-\left(x-\beta_{1}\right)^{\lambda}} d x\right]^{p}\right\}^{\frac{1}{p}}>k_{\lambda}(\sigma)\|F\|_{p, \varphi}, \\
& \left\{\int_{\beta_{1}}^{\infty}\left(x-\beta_{1}\right)^{-q \sigma-1}\left[\sum_{n=1}^{\infty} \frac{\ln \left[\left(n-\beta_{2}\right) /\left(x-\beta_{1}\right)\right] a_{n}}{\left(n-\beta_{2}\right)^{\lambda}-\left(x-\beta_{1}\right)^{\lambda}}\right]^{q} d x\right\}^{\frac{1}{q}}>k_{\lambda}(\sigma)\|a\|_{q, \psi} .
\end{aligned}
$$

In the same way, for $\delta=-1, \mu=\lambda-\sigma(>0)$ in Theorem 2 , setting $\varphi(x)=\left(x-\beta_{1}\right)^{p(1-\mu)-1}$, and $F(x)=\left(x-\beta_{1}\right)^{\lambda} f(x)$, we still can find some new equivalent reverse inequalities with the best possible constant factor.

\section{Competing interests}

The authors declare that they have no competing interests.

\section{Authors' contributions}

BY carried out the mathematical studies, participated in the sequence alignment and drafted the manuscript. AW participated in the design of the study and performed the numerical analysis. All authors read and approved the final manuscript.

\section{Acknowledgements}

This work is supported by the National Natural Science Foundation of China (No. 61370186) and 2013 Knowledge Construction Special Foundation Item of Guangdong Institution of Higher Learning College and University (No. 2013KJCX0140)

Received: 8 December 2014 Accepted: 25 February 2015 Published online: 05 March 2015

\section{References}

1. Hardy, GH, Littlewood, JE, Pólya, G: Inequalities. Cambridge University Press, Cambridge (1952)

2. Yang, B: On a more accurate Hardy-Hilbert's type inequality and its applications. Acta Math. Sin. 49(2), 363-368 (2006)

3. Mitrinović, DS, Pečarić, JE, Fink, AM: Inequalities Involving Functions and Their Integrals and Derivatives. Kluwer Academic, Boston (1991)

4. Pachpatte, BG: On some new inequalities similar to Hilbert's inequality. J. Math. Anal. Appl. 226, 166-179 (1998)

5. Gao, M, Yang, B: On the extended Hilbert's inequality. Proc. Am. Math. Soc. 126(3), 751-759 (1998) 
6. Hu, K: On Hardy-Littlewood-Pólya inequality. Acta Math. Sci. 20, 684-687 (2000)

7. Lu, Z: Some new generalizations of Hilbert's integral inequalities. Indian J. Pure Appl. Math. 33(5), 691-704 (2002)

8. Kuang, J, Debnath, L: On new generalizations of Hilbert's inequality and their applications. J. Math. Anal. Appl. 245, 248-265 (2000)

9. Sulaiman, WT: On Hardy-Hilbert's integral inequality. J. Inequal. Pure Appl. Math. 5(2), 25 (2004)

10. Zhong, W, Yang, B: A reverse Hilbert's type integral inequality with some parameters and the equivalent forms. Pure Appl. Math. 24(2), 401-407 (2008)

11. Yang, B: On a Hilbert-type operator with a symmetric homogeneous kernel of -1-order and applications. J. Inequal. Appl. 2007, Article ID 47812 (2007)

12. Zhong, J, Yang, B: On a relation to four basic Hilbert-type integral inequalities. Appl. Math. Sci. 4(19), $923-930$ (2010)

13. Yang, B: A mixed Hilbert-type inequality with a best constant factor. Int. J. Pure Appl. Math. 20(3), 319-328 (2005)

14. Yang, B: A half-discrete Hilbert's inequality. J. Guangdong Univ. Educ. 31(3), 1-8 (2011)

15. Xie, Z, Zeng, Z: A new half-discrete Hilbert's inequality with the homogeneous kernel of degree $-4 \mu$. J. Zhanjiang Norm. Coll. 32(6), 13-19 (2011)

16. Yang, B: A new half-discrete Mulholland-type inequality with parameters. Ann. Funct. Anal. 3(1), 142-150 (2012)

17. Chen, Q, Yang, B: On a more accurate half-discrete Mulholland's inequality and an extension. J. Inequal. Appl. 2012, Article ID 70 (2012). doi:10.1186/1029-242X-2012-70

18. Zhong, W: A mixed Hilbert-type inequality and its equivalent forms. J. Guangdong Univ. Educ. 31(5), 18-22 (2011)

19. Yang, B: The Norm of Operator and Hilbert-Type Inequalities. Science Press, Beijing (2009)

20. Kuang, J: Applied Inequalities. Shandong Science Technic Press, Jinan (2010)

21. Kuang, J: Real and Functional Analysis. Higher Education Press, Beijing (2002)

\section{Submit your manuscript to a SpringerOpen ${ }^{\ominus}$ journal and benefit from:}

- Convenient online submission

Rigorous peer review

- Immediate publication on acceptance

- Open access: articles freely available online

- High visibility within the field

- Retaining the copyright to your article 